\title{
Identification of ColR binding consensus and prediction of regulon of ColRS two-component system
}

\author{
Paula A Kivistik, Rait Kivi, Maia Kivisaar and Rita Hõrak*
}

\author{
Address: Estonian Biocentre and Institute of Molecular and Cell Biology, Tartu University, 51010 Tartu, Estonia \\ Email: Paula A Kivistik - paulaann@ut.ee; Rait Kivi - rait66@hotmail.com; Maia Kivisaar - maiak@ebc.ee; Rita Hõrak* - rhorak@ebc.ee \\ * Corresponding author
}

Published: 16 May 2009

BMC Molecular Biology 2009, 10:46 doi:10.1 186/1471-2199-10-46

This article is available from: http://www.biomedcentral.com/I47/-2/99//0/46

(C) 2009 Kivistik et al; licensee BioMed Central Ltd.

This is an Open Access article distributed under the terms of the Creative Commons Attribution License (http://creativecommons.org/licenses/by/2.0), which permits unrestricted use, distribution, and reproduction in any medium, provided the original work is properly cited.
Received: 18 November 2008

Accepted: 16 May 2009

\begin{abstract}
Background: Conserved two-component system ColRS of Pseudomonas genus has been implicated in several unrelated phenotypes. For instance, deficiency of $P$. putida ColRS system results in lowered phenol tolerance, hindrance of transposition of Tn4652 and lysis of a subpopulation of glucose-grown bacteria. In order to discover molecular mechanisms behind these phenotypes, we focused here on identification of downstream components of CoIRS signal transduction pathway.
\end{abstract}

Results: First, highly similar ColR binding sites were mapped upstream of outer membrane protein-encoding oprQ and a putative methyltransferase-encoding PP0903. These two ColR binding sequences were used as an input in computational genome-wide screening for new potential ColR recognition boxes upstream of different genes in P. putida. Biological relevance of a set of in silico predicted ColR-binding sites was analysed in vivo by studying the effect of ColR on transcription from promoters carrying these sites. This analysis disclosed seven novel genes of which six were positively and one negatively regulated by ColR. Interestingly, all promoters tested responded more significantly to the over-expression than to the absence of ColR suggesting that either ColR is limiting or ColS-activating signal is low under the conditions applied. The binding sites of CoIR in the promoters analysed were validated by gel mobility shift and/or DNase I footprinting assays. ColR binding consensus was defined according to seven ColR binding motifs mapped by DNase I protection assay and this consensus was used to predict minimal regulon of ColRS system.

Conclusion: Combined usage of experimental and computational approach enabled us to define the binding consensus for response regulator CoIR and to discover several new ColR-regulated genes. For instance, genes of outer membrane lipid A 3-O-deacylase PagL and cytoplasmic membrane diacylglycerol kinase DgkA are the members of ColR regulon. Furthermore, over 40 genes were predicted to be putatively controlled by CoIRS two-component system in $P$. putida. It is notable that many of ColR-regulated genes encode membrane-related products thus confirming the previously proposed role of CoIRS system in regulation of membrane functionality.

\section{Background}

Two-component signal systems are the main means for sensing the changing environment in a prokaryotic world
[1]. Typically, bacterial signal transduction systems consist of two components, a sensor histidine kinase and a response regulator. A specific compound or a physico- 
chemical property of the environment acts as a signal triggering the activation of a membrane embedded sensor, which in turn autophosphorylates and thereafter passes the signal to a response regulator via phosphoryl group transfer [2]. Phosphorylated response proteins mostly act as DNA binding transcription factors by activating or repressing the expression of target genes.

The number of genes for two-component proteins varies greatly between the genomes of sequenced bacteria, being for instance zero in case of Mycoplasma genitalium and 62 in a well-known model organism Escherichia coli [3]. The abundance of two-component systems seems to correlate with environmental and pathogenic versatility of a bacterium. Pseudomonas bacteria that colonise different habitats such as soil, water, plants and animal tissues, possess many two-component signal systems to cope with various environments. For example, over a hundreds genes encoding two-component system proteins are present in the genome of Pseudomonas aeruginosa [4].

The ColRS two-component signal transduction system, intrinsic to Pseudomonas species, consists of a sensor kinase ColS and a response regulator ColR. colRS operon is well-conserved among the sequenced members of Pseudomonas genus [5] suggesting that ColRS system could be important to these bacteria. ColRS pathway was first characterised in P. fluorescens as a system involved in the ability of bacteria to competitively colonise plant roots [6]. Our group observed a totally different function for ColRS system as transposition of Tn4652 was inhibited in phenol-starving colR- and colS-deficient P. putida [7]. Additionally, recently, we demonstrated that colR-deficient $P$. putida is sensitive to phenol [8] and displays a serious defect on solid glucose medium where a subpopulation of the mutant lyses [9]. ColRS system was also shown to be important in resistance of $P$. putida to divalent metal ions, especially $\mathrm{Mn}^{2+}[10]$. Importantly, the precise checkpoint of a response regulator ColR remained unclear in case of all these ColR-dependent phenotypes. However, two recent publications suggest that seemingly unrelated phenotypes of colR-deficient $P$. fluorescens and $P$. putida can most probably be explained by the compromised cell membranes of colR mutants. Namely, the first ColR-regulated genes that were identified in these two species, encoded different membrane functions. We demonstrated that oprQ and algD, which encode a porin protein and an exopolysaccharide alginate biosynthesis enzyme, respectively, are under the direct control of ColR in P. putida [8]. Concomitantly, de Weert et al [11] reported for $P$. fluorescens that an operon downstream of colRS hypothetically coding for membrane associated proteins, methyltransferase (orf222) and lipopolysaccharide kinase (inaA/ wapQ), is also regulated by ColR.
In spite of several recent studies disclosing the ColRS system as an important signal transduction pathway for pseudomonads, little is known about the downstream components of this signal system. Only two target genes of ColR have been identified in P. putida [8] and our unpublished data show that neither oprQ nor algD are involved in phenotypes characteristic to colR mutant. Thus, more ColR-regulated genes should be present in the genome of $P$. putida. In this study we aimed to determine the ColR binding consensus sequence and to use it in the screen of $P$. putida genome for the presence of new potential ColR binding sites, which would predict new ColR target genes and operons.

\section{Results \\ ColR (PP090I) regulates the expression of PP0903}

The expression of genes downstream of colRS operon is regulated by ColR in $P$. fluorescens [11]. This region of genome is well conserved among Pseudomonas bacteria [5] and therefore we presumed that the operon downstream of colRS (Fig. 1a) could be regulated by ColRS system in $P$. putida as well. This downstream operon consists of three genes (Fig. 1a). The first two, PP0903 coding for a putative methyltransferase and PP0904 coding for a lipopolysaccharide kinase InaA, are conserved among all 15 fully sequenced pseudomonads [5]. Putative orthologs of the third open reading frame PP0905 encoding for a hypothetical protein are present downstream of inaA in 13 sequenced members of Pseudomonas genus. In order to test whether these genes belong to ColR regulon in $P$. putida, we cloned the upstream region of PP0903 in front of lacZ gene in a promoter probe plasmid $\mathrm{p} 9 \mathrm{TT}_{\mathrm{B}} \mathrm{lac} Z$ and compared plasmid-encoded $\beta$-galactosidase activities in wildtype P. putida strain PaW85 and in its ColR-deficient derivative. Our unpublished results show that ColR-dependent phenotypes are more pronounced on solid medium if compared to that in liquid medium. Therefore, all enzyme activities presented in this study were measured from solid-medium-grown bacteria. The obtained data showed that ColR is a positive effector of PP0903 expression since $\beta$-galactosidase activity was comparable to the basal level of promoter probe vector $\mathrm{p} 9 \mathrm{TT}_{\mathrm{B}} \mathrm{lac} \mathrm{Z}$ in a colR-deficient strain but was 10-fold higher in a wild-type strain (Fig. 1b). As our previous study showed that ColR-regulated oprQ and $\lg \mathrm{g}$ promoters were affected by phenol [8], the $\beta$-galactosidase activity was also measured in the presence of phenol. Again, the activity of PP0903 promoter remained indistinguishable from the basal level of vector in the colR-mutant strain (Fig. 1b). However, phenol significantly enhanced the activity of PP0903 promoter in a wild-type strain where ColR is present (Fig. 1b). To confirm the role of ColR in the activation of PP0903 promoter, $\beta$-galactosidase measurements were also performed in colR-mutant derivative PaWRtaccolR where the expression of ColR was inducible from $\mathrm{P}_{t a c}$ promoter 
a

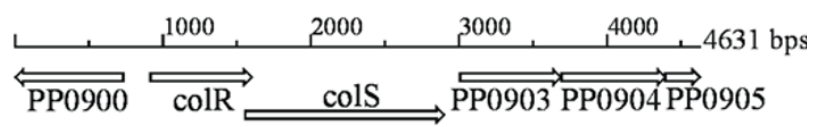

$\mathrm{b}$

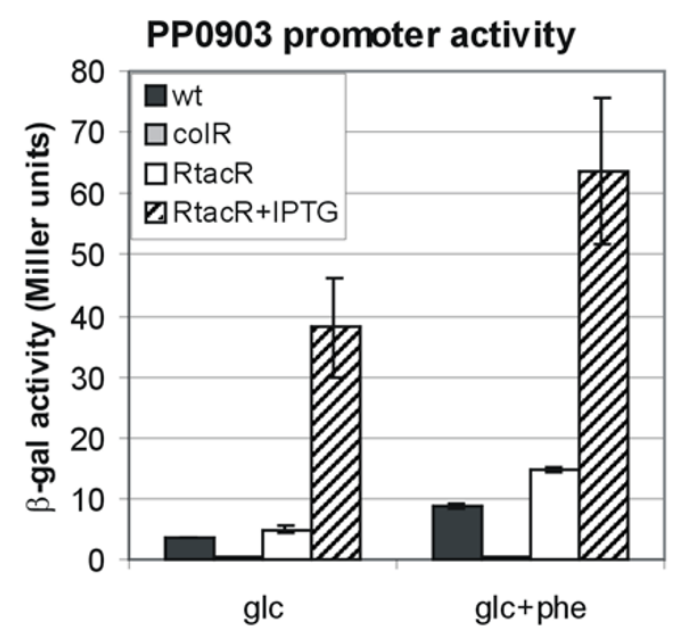

Figure I

ColR activates the expression of its downstreamlocating PP0903. (a) Organization of genes in P. putida colRS locus. (b) $\beta$-galactosidase ( $\beta$-gal) activities measured in $P$. putida wild-type strain PaW85 (wt), colR-defective strain PaWcolR (colR) and colR-defective strain PaWRtaccolR complemented with colR gene under the control of the inducible $\mathrm{P}_{\text {tac }}$ promoter (RtacR). All strains carried the reporter plasmid with the promoter of PP0903. ColR expression in strain PaWRtaccolR was induced with $0.5 \mathrm{mMIPTG}$ (RtacR+IPTG). Bacteria were grown either on glucose $(\mathrm{glc})$ or glucose plus $2.5 \mathrm{mM}$ phenol (glc+phe) minimal plates. Data (means and standard deviations) from at least three independent experiments are presented. For promoter probe vector

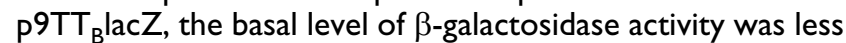
than 0.5 Miller units.

with addition of IPTG. The activity of PP0903 promoter was already recovered in PaWRtaccolR strain without the induction of colR (Fig. 1b). This was expected as immunoblot analysis of ColR expression had previously revealed significant leakiness of $\mathrm{P}_{t a c}$ promoter in that particular strain [7]. Over-expression of ColR resulted in a strong enhancement of PP0903 promoter over the wild-type level both on glucose and glucose plus phenol medium (Fig. 1b). Hence, response regulator ColR is indeed necessary for up-regulation of PP0903 promoter in P. putida. Strong induction of the promoter by ColR over-expression suggests that either the amount of ColR is limiting in the wild-type cells or the ColS-activating signal is low in tested conditions. Data also imply that the impact of ColR on the PP0903 activation becomes more prominent in bacteria experiencing phenol-caused stress.

\section{ColR binding sites in the promoter regions of oprQ (PP0268) and PP0903}

One possible way to find new target genes of a transcriptional regulator is to computationally analyze the whole genome of an organism. As a certain binding consensus of a transcription factor is needed for that, we aimed to specify the exact binding sites of ColR in the promoters under its control. Our previous paper demonstrated that purified histidine-tagged ColR bound in vitro to the promoters of $o p r Q$ and algD in a gel mobility shift assay [8]. Similar analysis with PP0903 promoter showed that ColR also directly interacts with this promoter DNA. In order to map the ColR binding sites we performed a DNase I footprinting assay to the DNAs of oprQ, algD and PP0903 promoter fragments labelled with $\mathrm{P}^{32}$. Fig. 2a illustrates the ColRrecognized regions in the promoters of oprQ and PP0903. ColR protected a 24-to-26-bp-long area in both promoters (Fig. 2b) depending on a strand analysed. ColR binding sites reside about 190 and 60 nucleotides upstream of translation start codons of oprQ and PP0903, respectively. The binding of ColR to PP0903 promoter resulted in the appearance of a hypersensitive site for DNase I just next to the ColR-protected region (Fig. 2a). Data also showed that the phosphorylated form of ColR (ColR P) binds to the DNA with higher affinity than the unphosphorylated ColR. However, the effect of phosphorylation was stronger in case of PP0903 compared to oprQ (Fig. 2a).

Despite the fact that ColR bound to the promoter DNA of algD in a gel mobility shift assay [8] we could not detect a ColR-binding site in this DNA by DNase I footprinting (data not shown). This suggests that the binding dynamics of response regulator ColR could be somewhat different if algD promoter is compared to the promoters of oprQ and PP0903. Most probably ColR recognition sequences in the $\operatorname{alg} D$ promoter diverge from ColR consensus (see Discussion).

\section{Computer search for potential ColR binding sites revealed several new ColR-regulated genes}

Comparison of ColR recognition sequences in the promoters of oprQ and PP0903 (Fig. 2b) shows clearly that these two sequences are similar. The most obvious common feature is a central T-track, 5-bp and 7-bp long in case of oprQ and PP0903, respectively (Fig. 2b). In total 10 identical nucleotides are present in these two ColR binding sites allowing to deduce a 13-bp-long consensus motif for ColR binding - ACGNNTTTTTNAC. High similarity of the two ColR binding boxes suggested that a genomewide computer search could reveal new potential target 
genes of ColR. Thus, sequences similar to identified ColR binding sites were searched from the genome of $P$. putida KT2440 using two free-access web-servers, PredictRegulon $[12,13]$ and Virtual Footprint $[14,15]$. Several inquiries with varying input sequence length and strand orientation were performed. Results of both programmes were compared and identical hits were chosen for experimental validation. Actually, the prediction and identification of new ColR binding sites presented below was a step-by-step process meaning that new predictions were made with every additional confirmed ColR binding site (see Methods). For experimental evaluation of the computer-predicted putative ColR-regulated promoters, they were cloned into a promoter probe plasmid $\mathrm{p} 9 \mathrm{TT}_{\mathrm{B}} \mathrm{lacZ}$ upstream of the lacZ gene. If a predicted ColR-binding site was located between two divergently transcribed genes, then this DNA region was cloned upstream of the reporter in both orientations (promoters of PP0035/0036, PP0900/0901 and PP2560/2561). $\beta$-galactosidase activities were measured in wild-type and ColR-deficient $P$. put$i d a$ cells grown overnight on glucose and glucose plus phenol solid media. Additionally, since promoter activity of PP0903 was strongly enhanced by over-expression of ColR (Fig. 1b) all promoters were tested also in a P. putida strain PaWRtaccolR where the expression of ColR can be induced from $\mathrm{P}_{\mathrm{tac}}$ promoter. Results of $\beta$-galactosidase assay show that promoters of PP0035, PP0036, PP0737, PP0900, PP1636, PP2560 and PP3766 are responsive to the absence and/or to the over-expression of ColR (Fig. 3). All promoters except PP0737 are positively affected by ColR. If promoters were tested in the presence of $2.5 \mathrm{mM}$ phenol then the effects of ColR-deficiency and overexpression became more evident in case of most promoters. For instance, while promoters of PP0036, PP0900, PP1636, PP2560 and PP3766 were only barely affected by the absence of ColR in the glucose-grown cells, then the impact of ColR upon these promoters became obvious in the glucose plus phenol-grown bacteria. It is also remarkable that all promoters analysed responded significantly more to the over-expression than to the absence of ColR. Namely, ColR over-expression clearly enhanced the activities of promoters that are under its positive control (Fig. 3). Especially drastic activation was seen in case of PP0035 where over-expressed ColR caused 30-fold increase in the promoter activity when compared to that in a wild-type strain (Fig 3). At the same time, the activity of PP0035 promoter was only 3-fold lower in glucose-grown colR mutant than in wild-type. Analogous to that, the overexpression of ColR lowered the activity of negatively ColR-affected PP0737 promoter about 3-fold but the lack of ColR affected this particular promoter less than 2-fold (Fig. 3). This data strongly suggest that either ColR amount is limiting in wild-type P. putida to affect significantly the expression of analysed target promoters or the signal perceived and transduced by ColS is low under the conditions employed by us.

It is important to point out that one new ColR-regulated gene, PP0900, locates just upstream of colRS genes and is transcribed divergently from PP0901 encoding colR (Fig. 1a). Thus, the putative ColR-binding site affecting the expression of PP0900 is actually located between PP0900 and colR. Analysis of this particular promoter in the direction of colR (PP0901) did not reveal clear ColR-responsiveness under any conditions examined (Fig. 3). Therefore we conclude that ColR does not auto-regulate its own expression.

\section{ColR binding sites in the promoters of predicted target genes}

We aimed to confirm that in silico predicted ColR binding sites upstream of different genes, which expression was ColR-dependent in vivo, could really bind ColR. For that purpose, we performed a DNase I footprinting analysis to six promoter regions that were tested in a $\beta$-galactosidase assay (Fig. 3). Figure 4a shows ColR protection patterns of five different promoter regions - PP0035/0036, PP0737, PP0900/0901, PP2560/2561 and PP3766. The length of ColR-protected area varied between the promoters analysed ranging from 18-bp in one strand of PP0737 up to 46-bp in PP3766 (Fig. 4b). Phosphorylation of ColR by ColS significantly increased its affinity to DNAs upstream of PP0900/0901, PP2560/2561 and PP3766. The binding of ColR to several promoter DNAs changed the DNase I digestion pattern revealing new hypersensitive sites for DNase. Paradoxically, ColR could not affect the DNase I digestion pattern of the PP1636 promoter (data not shown), although, this promoter was influenced by ColR in vivo (Fig. 3) and ColR binding to this DNA was clearly detectable in a gel mobility shift assay (Fig. 5). It is noteworthy that the same was seen in case of ColR-regulated algD promoter (see above). Thus, performed footprint analysis could not reveal all ColR binding sites in the promoters of its target genes. Nevertheless, data presented in Fig. 4 verifies that ColR indeed binds to the computationally predicted sites in five new ColR-responsive promoters.

\section{ColR binding consensus and potential regulon}

The sequences of seven ColR binding sites identified by DNase I footprinting analysis (Fig. 2 and Fig. 4) were aligned to define a more precise ColR binding consensus than initial one, which was deduced only from two binding motifs detected in the promoters of oprQ and PP0903. The length of the region protected by ColR varied significantly in different promoters (Fig. 2 and Fig. 4). Therefore, rather long DNA fragments (presented in Table 1) were compared in order to find out whether sequences surrounding the core ColR-recognition site are conserved as 
a

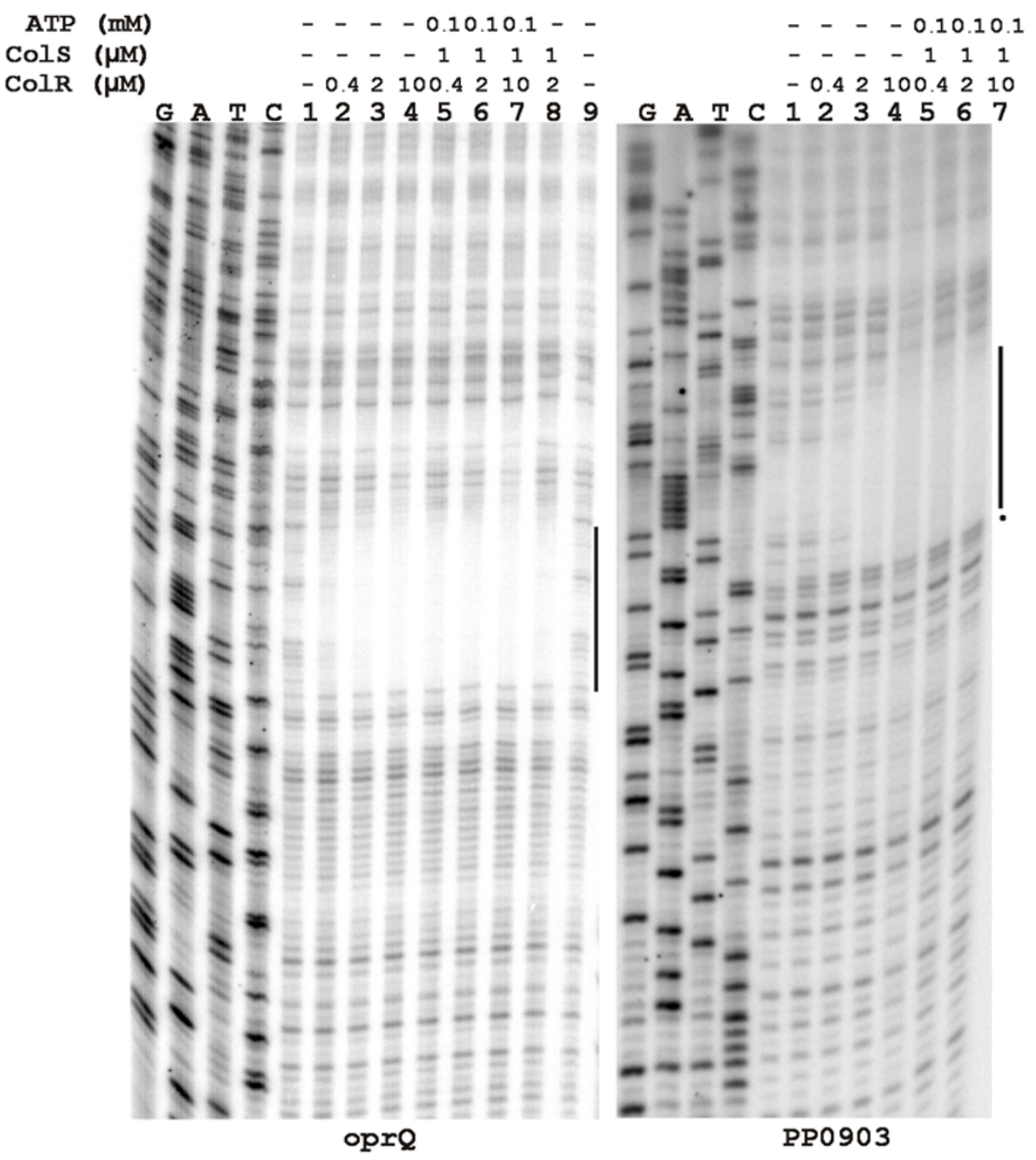

b

oprQ

CAACCTGCATTTCACGACTTTTTGAC 196 bp > ATG

CGTAAAGTGCTGAAAAACTGTAAA 192 bp > TAC

PP0903

GTGCCCAACGTTTTTTTCACATTGGC 60 bp > ATG

CCACGGGTTGCAAAAAAAGTGTAAC 63 bp > TAC

\section{Figure 2}

ColR binding sites in the promoter regions of oprQ and PP0903. (a) DNase I footprinting assay performed on oprQ and PP0903 promoter DNA. Dideoxy-chain termination sequences corresponding to the probes are shown in the order of G, A, T and C. Concentrations of DNA-binding protein CoIR, its cognate kinase ColS, and phosphoryl-donor ATP are shown above each lane. Vertical black lines indicate ColR-protected regions and dot represents a site hypersensitive to DNase I cleavage. (b) Sequences of CoIR-protected regions in the promoters of oprQ and PP0903. A site hypersensitive to DNase I cleavage in the presence of ColR is in bold. Nucleotides identical in two ColR binding sites are underlined. Distance of the ColR-binding site from the start codon is presented. 

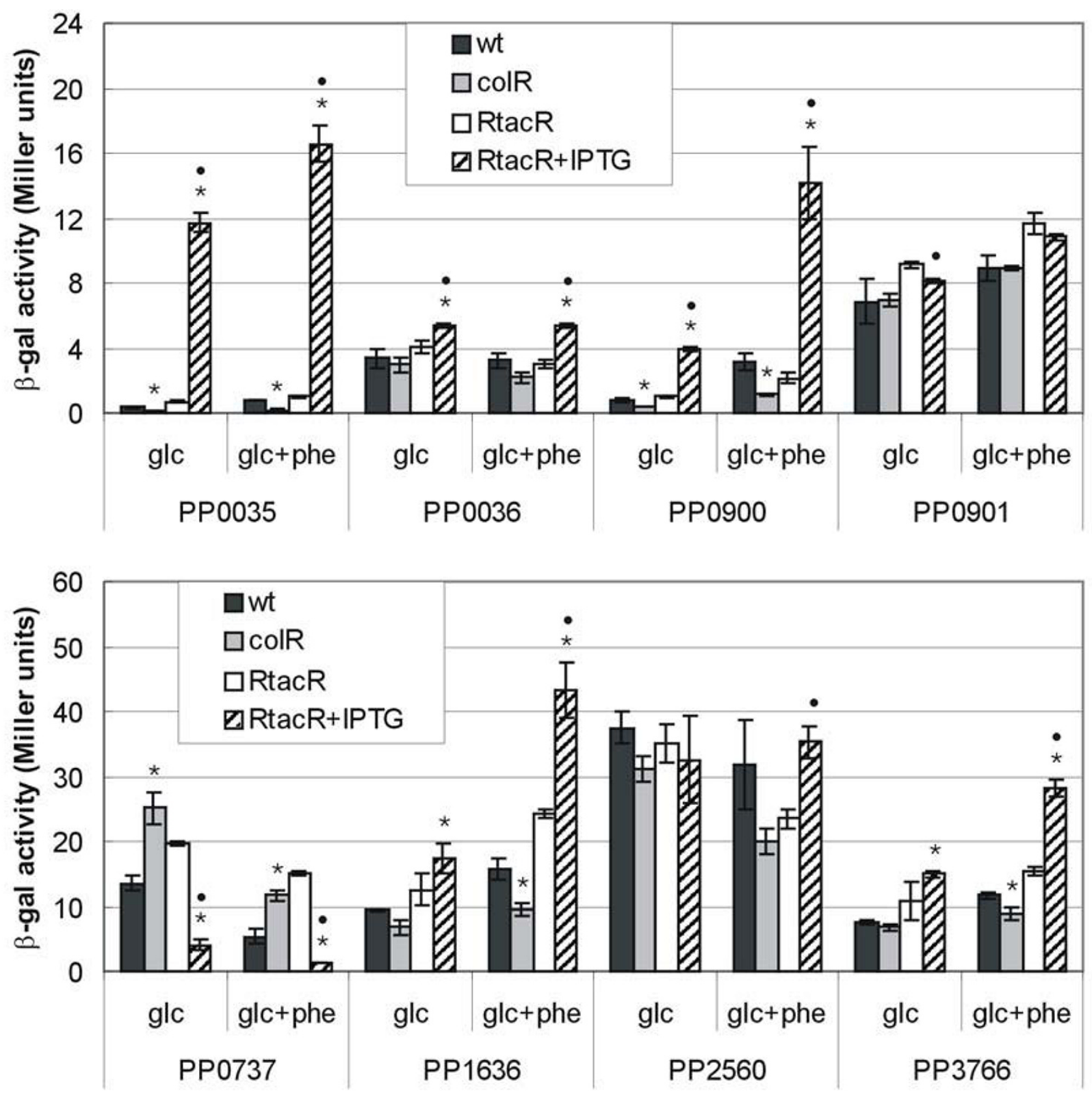

Figure 3

Effects of ColR on the expression of a set of computationally predicted promoters. $\beta$-galactosidase ( $\beta$-gal) activities measured in wild-type (wt), colR-defective (colR) and colR-defective strain PaWRtaccolR complemented with colR gene under the control of the $P_{\text {tac }}$ promoter (RtacR). P. putida strains carried reporter plasmids with promoter regions of following genes: PP0035, PP0036, PP0900, PP090 I, PP0737, PPI636, PP2560 or PP3766. Bacteria were grown either on glucose (glc) or glucose plus $2.5 \mathrm{mM}$ phenol (glc+phe) minimal plates. ColR expression in strain PaWRtaccolR was induced with $0.5 \mathrm{mM}$ IPTG (RtacR+IPTG). Data (means and standard deviations) from at least three independent experiments are presented. For promoter probe vector $\mathrm{P} \mathrm{TT}_{\mathrm{B}}$ lac $Z$, the basal level of $\beta$-galactosidase activity was less than 0.5 Miller units. Asterisks above the bars indicate statistically significant differences $(p<0.05$ according to t-test) between the promoter activities of particular strain and wild-type. Dots indicate significant differences between PaWRtaccolR strain and PaWRtaccolR strain grown with IPTG. 
well. Fig. 6 demonstrates a ColR binding consensus created using WebLogo server [16]. In the middle of the sequence a well-conserved 16-bp-long core with 8 totally conserved positions is present, whereas most of the distant positions are much less conserved. Therefore, only the core region was defined as a final ColR binding consensus $((\mathrm{T} / \mathrm{C})(\mathrm{T} / \mathrm{C}) \mathrm{NA}(\mathrm{C} / \mathrm{G}) \mathrm{NN}(\mathrm{T} / \mathrm{C}) \mathrm{TTTTT}(\mathrm{C} / \mathrm{G}) \mathrm{AC})$. Next, this 16-bp-long core was used to predict minimal regulon of ColRS system. 41 ColR binding motif-resembling sites were extracted from $P$. putida genome by searching the upstream regions (500 bp upstream of the translation start) of all genes using a PredictRegulon server tool. As several binding sites locate between divergently transcribed genes one may assume that ColR regulon potentially consists of more than 50 different genes (see Additional file 1). However, whilst the first 11 sites of prediction match perfectly with 7 input sequences, the rest of the matches score lower than the cut-off value ( 6.76929 in our prediction; see Additional file 1). Therefore, they should be considered as potential novel ColR sites, which should be confirmed experimentally. It is notable that three genes (PP1692, PP2322 and PP2323) predicted as ColR-regulated possess two potential ColR-binding sites in their promoter regions (Additional file 1). There are genes of different functions in the predicted regulon of ColRS two-component system (Additional file 1), but in accordance with previous results, this signal system seems to regulate many membrane associated functions. Namely, $56 \%$ of putative ColR binding sites locate upstream of genes implicated in various membrane functions. Furthermore, inspection of the first 26 sites shows that even $87 \%$ of them locate upstream of genes coding for membrane proteins (Additional file 1). It is also remarkable that about one third of the putative members of ColR regulon code for hypothetical proteins (Additional file 1).

\section{Discussion}

Two-component signal transduction systems reside at the top of regulatory cascades. Therefore, to decipher the role of a particular two-component pathway it is crucial to specify the downstream components of the cascade. This study focuses on identification of target genes of a Pseudomonas two-component system ColRS, thus serving as an indispensable step in the way of unravelling the mechanisms that trigger different phenotypes related to ColRS deficiency.

Previously, we have searched for ColR-regulated genes by using a promoter library created from total chromosomal DNA of P. putida [8]. This screen disclosed only two ColR target genes, thus forcing us to try different approach to acquire more information about the putative regulon of ColRS system. We aimed to define a binding consensus for the transcription factor ColR and to search for similar sequences in the upstream regions of genes in the genome of $P$. putida. Seven experimentally verified ColR binding sites demonstrated a highly conserved ColR-binding motif with 8 fully conserved nucleotides in the 16-bplong core binding box (Fig. 6). The binding motifs of OmpR subfamily response regulators, among whom ColR belongs http://www.pseudomonas.com, consist typically of direct repeats separated by four to five nucleotides [17]. Careful inspection of ColR binding sites reveals that there are also two direct repeats. Although one of the repeats is much less conserved than the other, such structure of the binding site indicates that ColR binds to the DNA as a dimer. Notably, our data suggest that in addition to the highly conserved core sequence, ColR may also recognize and bind to less conserved sites. Namely, ColR regulates algD promoter in vivo and binds to this promoter in a phosphorylation-dependent manner according to gel mobility shift experiments [8]. Yet, computational analysis could not find a good ColR binding motif and DNase I protection analysis did not locate ColR binding site in the promoter region of algD. Nevertheless, the sequence upstream of algD contains many T-rich tracks characteristic for ColR binding consensus. If we lowered the specificity of Virtual Footprint prediction by allowing three mismatches in the input consensus then four putative ColR binding sites were found in algD promoter region, which was previously shown to bind ColR in DNA shift assay (data not shown). Probably ColR binds to these less conserved and thereby with lower affinity sites, but DNA cleavage by DNase I destabilizes the ColR-DNA complex and hence the ColR protected area cannot be detected by DNase footprint assay. Analogously, the nucleoprotein complex between the ColR and the upstream region of PP1636 probably dissociates due to DNase I cleavagecaused destabilization.

Given that some promoter regions (e.g. those of PP1692 and PP2322/2323) contain putatively more than one ColR consensus-resembling site (Additional file 1) one may hypothesise that co-operative binding of ColR to several sites may be necessary for regulation of these ColR target promoters. In this connection it is interesting to note that in silico analysis of seven experimentally verified ColR-binding promoters, revealed more putative ColR boxes than identified by DNase footprint assay. Namely, promoter regions of oprQ, PP0900/0901 and PP2560/ 2561 contain additional less-conserved ColR boxes (data not shown). Further experiments should prove whether these additional sites really support ColR binding to these promoters.

In this study several new ColR-dependent promoters were identified. Intriguingly, the effect of ColR absence upon promoter activities was rather modest (PP0035, PP0900, PP0737, PP1636) or not detectable (PP0036, PP2560, 
a

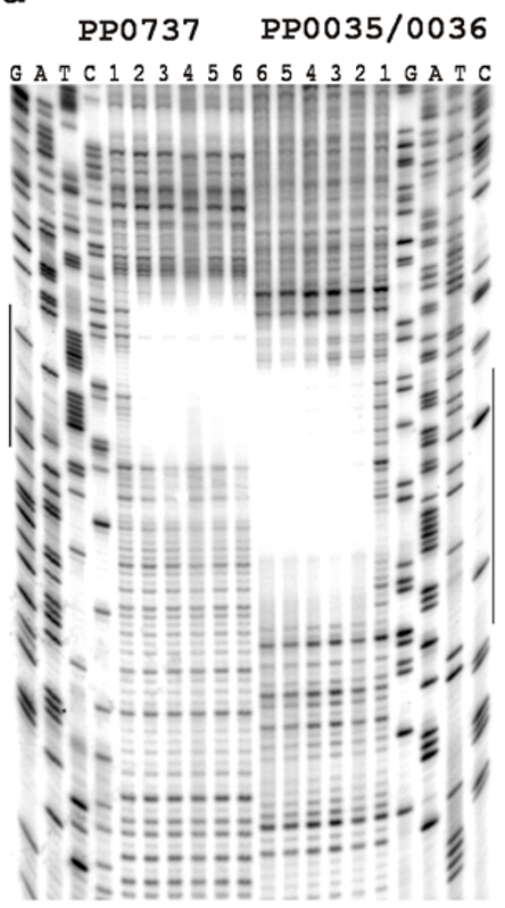

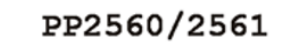

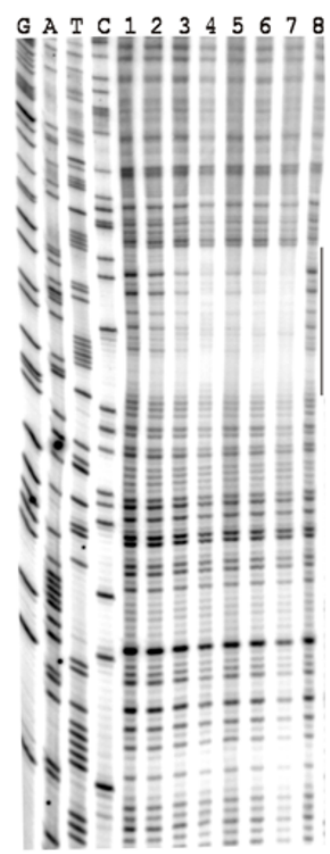

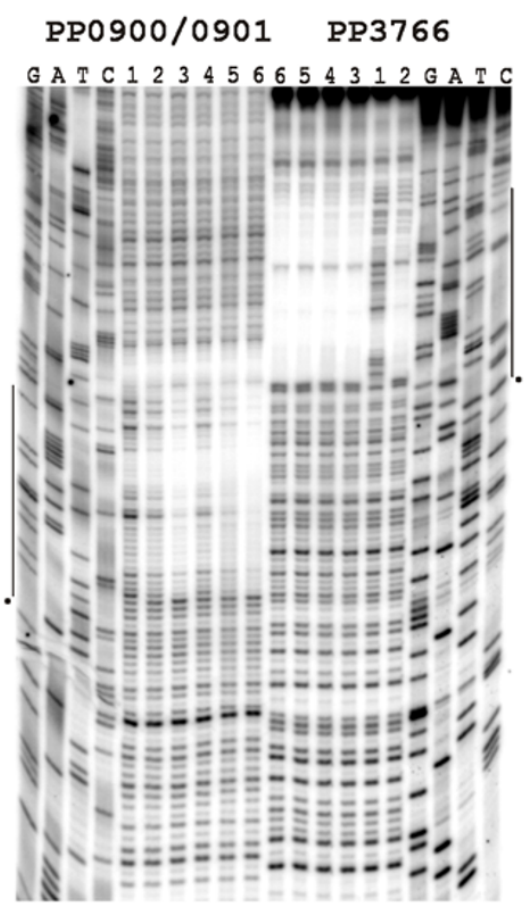

b

$\begin{array}{ll}\text { PP0737 } & \\ \text { CGTTGTGAAAAAAACGTG } & 86 \mathrm{bp}>A T G \\ \text { CAACACTTTTTTTGCACTTTTTTCCGA } & 75 \mathrm{bp}>T A C\end{array}$

PP0035/0036

AGGTGAAAAAATAGTGAACTAAATGTAAATGCGTAATTAGCC $195 \mathrm{bp}>$ ATG (of PP0036) GTTTCCACTTTTTTATCACTTGATTTACATTTACGCATT $201 \mathrm{bp}>T A C$

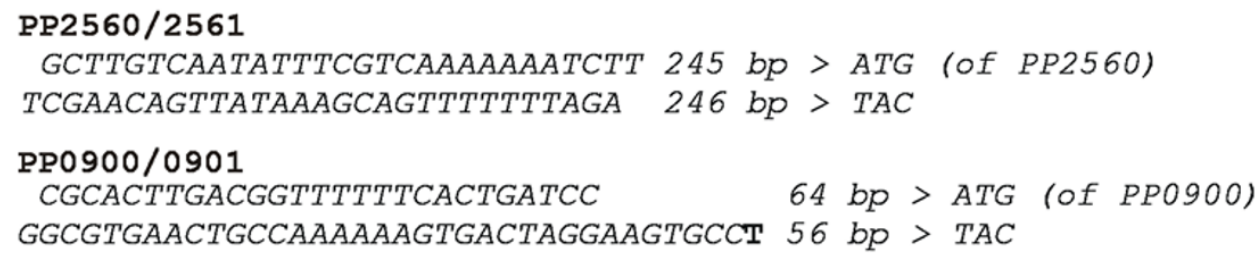

Figure 4

ColR-protected regions in the promoters of PP0737, PP0035/0036, PP2560/256I, PP0900/090I and PP3766. (a) DNase I footprinting assay performed on promoter DNAs of PP0737, PP0035/0036, PP2560/256I, PP0900/090I and PP3766. Lane I is always a DNase I-only ladder, following lanes represent different concentrations of DNA-binding protein ColR in a non-phosphorylated (lanes 2 and 3; in case of PP2560/256I lanes 2 to 4) and phosphorylated state (lanes 4 to 6; in case of PP2560/256I lanes 5 to 7). Concentrations of ColR in reactions with ColS and ATP were $0.6 \mu M, 3 \mu M$ and I5 $\mu M$ if DNA upstream of PP3766, PP0900/090I or PP2560/256I was used as a binding target, but $0.5 \mu \mathrm{M}, 2.5 \mu \mathrm{M}$ and I2.5 $\mu \mathrm{M}$ in case of PP0737 and PP0035/0036 promoter DNA. Concentration of ColR-phosphorylating ColS was always I $\mu$ M and ATP was 0.1 $\mathrm{mM}$. Only the two higher concentrations of ColR are used in the binding reactions with non-phosphorylated CoIR (lanes 2 and 3), except for PP2560/256I where all three CoIR concentrations are presented (lanes 2 to 4). (b) Sequences of the ColR-protected regions in the promoters of PP0737, PP0035/0036, PP2560/256I, PP0900/090 I and PP3766. Distance from the start codon is shown and the sites hypersensitive to DNase I cleavage in the presence of ColR are highlighted in bold. 
PP3766) when bacteria grew on glucose medium (Fig. 3). The greatest effect was seen in case of PP0903 promoter whose activity was 10 -fold down-regulated in a ColR-deficient $P$. putida (Fig. 1). However, the role of ColR in regulation of its target genes was confirmed by analysis of promoter activities under conditions of ColR over-expression. Indeed, most promoters tested were strongly influenced by ColR over-expression, especially when phenol was present in the growth medium (Fig. 1 and Fig. 3). This data indicate that the amount of transcriptionally active ColR is not sufficient enough to influence significantly most of its target promoters in wild-type P. putida in our assay conditions. Given that the phosphorylated form of ColR bound to its recognition sequences more avidly it is reasonable to conclude that actually the phosphorylated form of ColR is limiting in bacteria growing on glucose solid medium. Therefore, we consider that most probably the ColS-activating signal is low under our assay conditions. ColR over-expression could apparently mimic the conditions evoked by the signal and therefore the ColRdependence of its target promoters was seen more clearly in the situation of ColR over-expression. This scenario is in good accordance with our previous data showing that over-expression of ColR can compensate the defect in signal transfer from ColS to ColR when the participation of ColRS system in regulation of Tn4652 transposition was examined [7]. The signal sensed by ColS is not known so far. However, our observation that the impact of ColR on the expression of its target promoters was greater in the

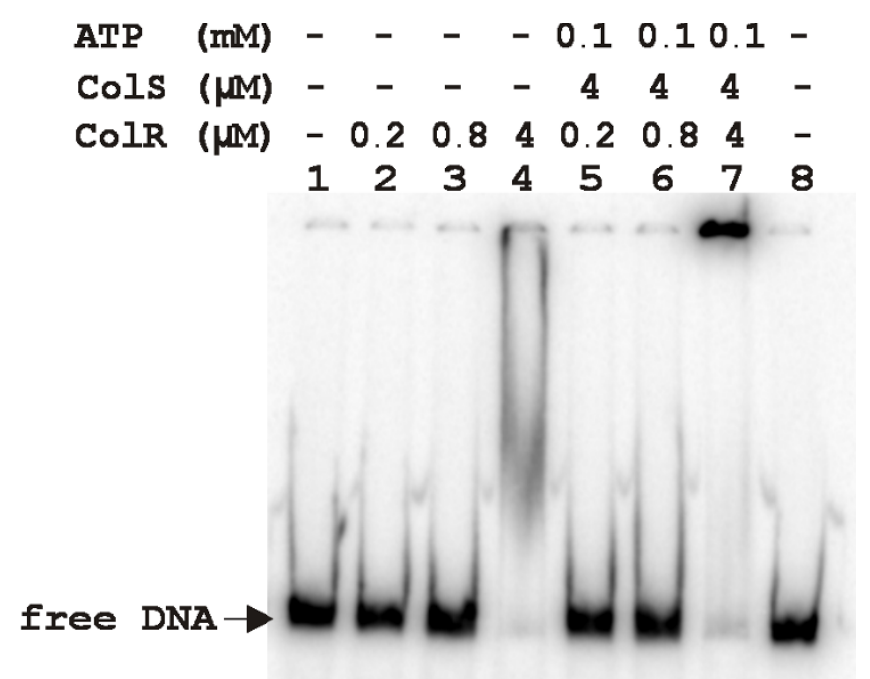

Figure 5

Gel mobility shift assay of the promoter DNA of PP I 636. Approximately $0.5 \mathrm{ng}$ ( $1000 \mathrm{cpm})$ of the DNA probe was incubated with different amounts of histidinetagged purified CoIR (lanes 2 to 4). To test the effect of phosphorylation on CoIR binding activity, ColS and ATP were added to the reactions (lanes 5 to 7 ). presence of phenol (Fig. 1 and Fig. 3) indicates that phenol-caused stress could be one of the conditions where ColRS signal transduction pathway is, at least partially, activated. This suggestion agrees with our previous results demonstrating the participation of ColRS system in phenol tolerance of $P$. putida [8].

Current study shows that ColR regulates directly genes locating upstream and downstream of its own gene. Like in $P$. fluorescens, ColR of $P$. putida also activates the downstream of colRS locating operon, that was suggested to be involved in fine-tuning of the outer membrane permeability [11]. It is notable that ColR regulates several other outer membrane protein-encoding genes confirming the role of ColRS system in the regulation of membrane functionality $[8,9,11]$. In addition to our previous finding that outer membrane protein-encoding oprQ is negatively controlled by ColR [8], the current study revealed that ColR is a negative factor also for PP0737 which codes for a protein orthologous to lipid A 3-O-deacylase PagL in P. aeruginosa [5]. The outer membrane-locating PagL modifies lipopolysaccharides by deacylation of lipid A at 3-O-position $[18,19]$. PagL becomes important under specific conditions only, e.g., it will be needed for mutants deficient in aminoarabinose-modified lipid A to resist cationic antimicrobial peptides [20]. In pathogenic Gram-negative bacteria the PagL-dependent deacylation of lipid A reduces the ability of lipid A to activate the Toll-like receptor 4 of the host, thus helping pathogens to avoid innate immune recognition [21]. The role of PagL has not been studied in P. putida so far.

Our data show that besides affecting the composition of outer membrane, ColR also regulates other membrane compartments. For instance, ColR activated PP0900 and PP1636 coding for two cytoplasmic membrane-locating enzymes, putative type 2 phosphatidic acid phosphatase (PAP2) and diacylglycerol kinase DgkA, respectively. These two enzymes most probably affect the membrane lipid homeostasis as they reversely regulate the abundance of phosphatidic acid and diacylglycerol, the precursor of phospholipid synthesis and the by-product of the synthesis of membrane-derived oligosaccharides, respectively [22]. Given that prediction of ColR regulon revealed another fatty acid and phospholipid metabolism related gene acpP (PP1915), coding for acyl carrier protein, as a putative ColR target gene, indeed, ColRS system may be involved in phospholipids homeostasis.

Since ColR is highly conserved among all Pseudomonas species [5] it was reasonable to presume that ColR-binding sites may also be similar in pseudomonads. Genomewide prediction of potential ColR-binding sites in $P$. fluorescens $\mathrm{PfO}-1$ disclosed seven genes that could be members of ColR regulon both in P. putida and $P$. fluorescens 


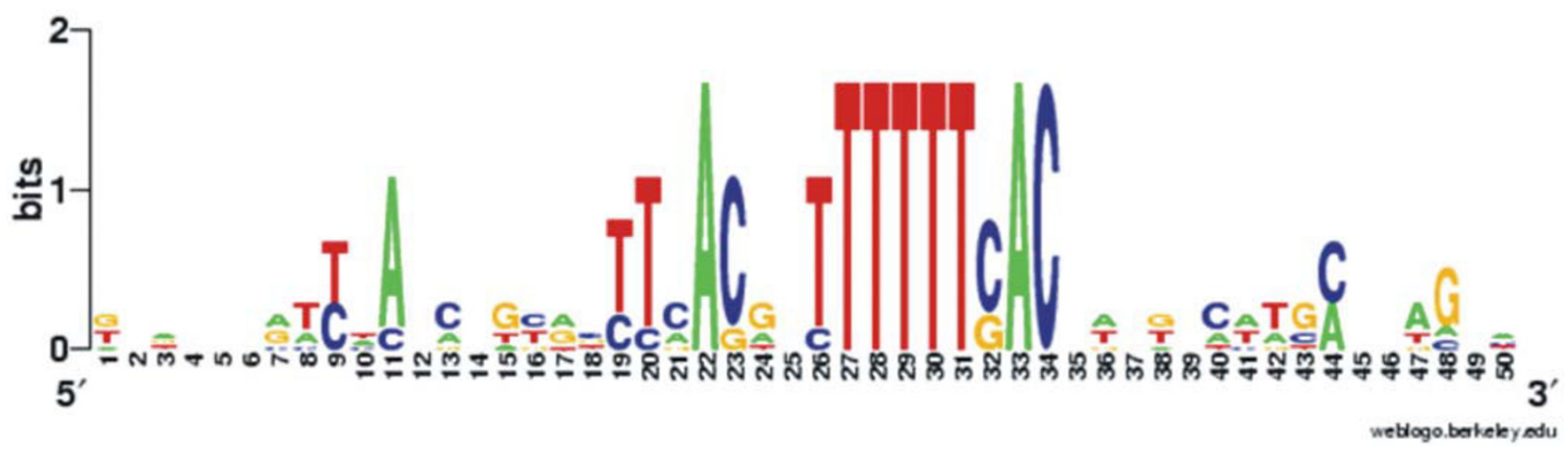

\section{Figure 6}

ColR binding consensus. Response regulator CoIR binding consensus sequence created with WebLogo server (I6). Sequence logo was drawn from 7 different ColR binding sites (Table I) identified upstream of PP0035/0036, oprQ (PP0268), PP0737, PP0900, PP0903, PP2560/256I, and PP3766. The degree of sequence conservation at each position is indicated as a total height of a stack of letters, measured in arbitrary "bit" units, with two bits possible at each position.

(see Additional file 2). Namely, ColR-binding box was found upstream of $P$. fluorescens $\mathrm{PfO}-1$ genes orthologous to P. putida dgkA-1, colR/PP0900, PP0737, PP0903, PP1058, PP1692 and PP5152 (data not shown). Additional screening of $P$. aeruginosa PAO1, P. syringae tomato DC3000 and $P$. syringae phaseolicola 1448A revealed that all these organisms contain perfect ColR-recognition sites upstream of $d g k A-1$ orthologs. Therefore, it is highly possible that $d g k A$ is a member of ColR regulon in all these pseudomonads.

\section{Conclusion}

Current study identified a 16-bp-long binding consensus of response regulator ColR ((T/C)(T/C)NA(C/G)NN(T/
C)TTTTT(C/G)AC), which helped us to discover new genes controlled by ColRS two-component system in $P$. putida. Notably, several new ColR target genes (PP0035, PP0737, PP0900, PP1636 and PP2560) code for different membrane proteins supporting our previous assumption that the primary target of ColRS two-component system is the cell membrane. Regulon prediction suggests that ColR could regulate over 40 genes and many of them code for membrane-associated functions as well. However, it is not clear yet which of the target genes are responsible for specific ColR-related phenotypes such as lowered phenol tolerance, hindrance of transposition of $\operatorname{Tn} 4652$ and glucoseinduced lysis of a subpopulation of the colR mutant [7-9]. Considering the number of ColR regulon genes it is highly

Table I: Seven sequences that were used for creating ColR binding consensus

\begin{tabular}{lll}
\hline Sequence $^{\mathbf{a}}$ & Locus/Gene & Product \\
\hline $\begin{array}{l}\text { ACGATAGTTCAGCCTTTTTTCACGTTTTTTTCACAACG- } \\
\text { CAAAGCTTTGA }\end{array}$ & PP0737 & conserved hypothetical protein \\
\hline $\begin{array}{l}\text { TGAAATGCCCACATAGGCTTCACACTTTTTTCACAAGGACT- } \\
\text { GGCTCAGTA }\end{array}$ & PP3766/gloA & lactoylglutathione lyase \\
\hline $\begin{array}{l}\text { TTACGCATTTACATTTAGTTCACTATTTTTTCACCTTTGCCTC- } \\
\text { CATAGGC }\end{array}$ & PP0035 & GtrA family protein \\
\hline $\begin{array}{l}\text { GGGGAAATTCACCGCACTTGACGGTTTTTTCACTGATCCT- } \\
\text { TCACGGACA }\end{array}$ & PP0900 & PAP2 family protein \\
\hline $\begin{array}{l}\text { GCATCGCATTATCAGCGGCTAAGATTTTTTTGACGAAATATT- } \\
\text { GACAAGCT }\end{array}$ & PP256I & secreted hemolysin-type calcium-binding bacteriocin, putative \\
\hline $\begin{array}{l}\text { GGTTATATCAACCTGCATTTCACGACTTTTTGACATTTG- } \\
\text { TAATCGCACAC }\end{array}$ & PP0267/oprQ & outer membrane protein OprE3 \\
\hline $\begin{array}{l}\text { TATCAGGTCAACGGGTGCCCAACGTTTTTTTCACATTGGCAT- } \\
\text { GACCTGAT }\end{array}$ & PP0903 & conserved hypothetical protein
\end{tabular}

a Most conserved 16-bp-long sequences that were used as input in the final regulon prediction are indicated in bold 
possible that not one but several ColR target genes are involved in the formation of above-mentioned phenotypes. Given that ColRS two-component system is regulating several membrane-related genes, our further experiments are directed towards clarification the role of ColR target genes in the membrane functionality.

\section{Methods}

\section{Bacterial strains, plasmids and media}

P. putida strain PaW85 [23], which is isogenic to fully sequenced KT2440 [24], its colR-deficient derivative PaWcolR [7] and PaWcolR derivative strain PaWRtaccolR capable of over-expression of ColR [7] were used in this study. E. coli strain DH5 $\alpha$ (Invitrogen) was used for DNA manipulations. Bacteria were grown in Luria-Bertani (LB) medium [25] or in M9 minimal medium [26] containing $10 \mathrm{mM}$ glucose. Concentration of phenol was $2.5 \mathrm{mM}$ in minimal medium. When necessary, the growth medium was supplemented with ampicillin $\left(100 \mu \mathrm{g} \mathrm{ml}^{-1}\right)$ or chloramphenicol $\left(20 \mu \mathrm{g} \mathrm{ml}^{-1}\right)$ for Escherichia coli and with benzylpenicillin $\left(1,500 \mu \mathrm{g} \mathrm{ml}^{-1}\right)$, chloramphenicol $(300 \mu \mathrm{g}$ $\left.\mathrm{ml}^{-1}\right)$ or kanamycin $\left(50 \mu \mathrm{g} \mathrm{ml}^{-1}\right)$ for $P$. putida. X-gal (5bromo-4-chloro-3-indolyl-beta-D-galactopyranoside)

( $75 \mu \mathrm{g} \mathrm{ml}^{-1}$ ) was added to the growth medium for visual evaluation of promoter activities. $0.5 \mathrm{mM}$ IPTG (isopropyl $\beta$-D-1-thiogalactopyranoside) was used to induce $\mathrm{P}_{\text {tac }}$ promoter. $P$. putida was incubated at $30^{\circ} \mathrm{C}$ and $E$. coli at $37^{\circ} \mathrm{C}$. E. coli and P. putida cells were electrotransformed according to the protocol of Sharma and Schimke [27].

\section{Construction of reporter plasmids}

For cloning of different promoter regions into promoter probe plasmid $\mathrm{p} 9 \mathrm{TT}_{\mathrm{B}} \mathrm{lacZ}$ [8] the PCR-amplified DNA fragments were used. Restriction site-containing oligonucleotides used in the process are listed in Additional file 3. Different promoter regions were amplified by PCR using the purified chromosomal DNA of $P$. putida PaW85 as a template. PCR fragments were restricted with the appropriate enzyme (Additional file 3 ) and cloned upstream of lacZ gene in the plasmid $\mathrm{p} 9 \mathrm{TT}_{\mathrm{B}}$ lacZ. Orientation of a promoter fragment was verified by PCR.

\section{Enzyme assay}

All enzyme activities presented in this paper were measured from solid-medium-grown bacteria. Bacteria grown both on glucose or glucose plus $2.5 \mathrm{mM}$ phenol containing M9 minimal medium were scraped off from the plates using toothpicks and suspended in M9 solution. For one suspension 24-hours-grown bacteria were collected from a sector comprising approximately one-twelfth of the Petri plate. $\beta$-galactosidase activity was assayed according to a previously described protocol [28].

\section{DNA gel mobility shift assay}

ColR and N-terminally truncated ColS used in DNA gel mobility shift assay, were over-expressed and purified as
His-tagged proteins by published protocol [7]. Oligonucleotides used in PCR to generate DNA probes are listed in Additional file 3. Gel mobility shift assay was performed according to a previously described protocol [8].

\section{DNase I footprinting assay}

DNA fragments for DNase I footprinting assay were amplified from the purified chromosomal DNA of $P$. putida PaW85 by PCR. Oligonucleotides used to generate DNA probes by PCR are listed in Additional file 3. One oligonucleotide was end-labelled by phosphorylation with $\left[\alpha{ }^{32} \mathrm{P}\right]$-ATP and thus PCR reactions created products with specific labelling of one DNA strand. The labelled DNA fragments were purified by native $5 \%$ polyacrylamide gel electrophoresis, eluted (buffer containing $0.5 \mathrm{M}$ $\mathrm{NH}_{4} \mathrm{Ac}, 10 \mathrm{mM}$ MgAc, $1 \mathrm{mM}$ EDTA and $0.1 \%$ SDS) and resuspended in water. For the binding reaction, different amounts of purified $P$. putida his-tagged ColR protein (concentrations of ColR are specified in the text as they varied in case of different DNA probes) were combined with 30000 c.p.m. of labelled DNA fragment, $25 \mathrm{mM}$ Tris-HCl (pH 7.5), $10 \mathrm{mM} \mathrm{MgCl}_{2}, 1 \mathrm{mM} \mathrm{CaCl}_{2}, 0.1 \mathrm{mM}$ EDTA, $50 \mathrm{mM} \mathrm{KCl}, 5 \mu \mathrm{g}$ of BSA, $5 \mu \mathrm{g}$ of salmon sperm DNA and 5\% glycerol in a final volume of $100 \mu \mathrm{l}$. Reactions with different volume of proteins were equalized with the addition of appropriate amount of ColR storage buffer. To test the binding of phosphorylated ColR with the DNA probes, ColS was first autophosphorylated by incubation in the presence of $0.1 \mathrm{mM}$ ATP in the reaction buffer for $15 \mathrm{~min}$. After addition of ColR to the phosphorylated ColS and further incubation for $15 \mathrm{~min}$, labelled DNA was added to the reaction mixture. ColR was allowed to bind to DNA during $20 \mathrm{~min}$ at room temperature before the start of digestion by DNase I ( $0.25 \mathrm{U}$, Fermentas) for $3 \mathrm{~min}$. Reactions were stopped by the addition of $100 \mu \mathrm{l}$ of a solution containing 0.1 M EDTA, $0.1 \%$ sodium dodecyl sulphate, $1.6 \mathrm{M}$ ammonium acetate and $20 \mu \mathrm{g}$ of sonicated salmon sperm DNA per ml. The footprinting reaction mixtures were subsequently extracted once with phenol and chloroform $(1: 1 \mathrm{v} / \mathrm{v})$ and once with chloroform and, finally, the DNA was precipitated with ethanol. The DNA fragments were resuspended in $7 \mu \mathrm{l}$ of sequence loading buffer (deionized formamide containing $10 \mathrm{mM}$ EDTA, $0.3 \%$ bromophenol blue and $0.3 \%$ xylene cyanol) and loaded onto a $6.5 \%$ polyacrylamide gel that contained $8 \mathrm{M}$ urea. DNA sequencing reactions were performed with a Sequenase version 2.0 kit (US Biochemicals) and were loaded on a sequencing gel as size markers. After the run, the gels were dried and exposed to a PhosphorImager screen (Amersham Biosciences).

\section{In silico identification of putative ColR binding sites}

Putative ColR binding sites in the genomes of $P$. putida KT2440,P. aeruginosa PAO1, P. fluorescens PfO-1, P. syringae tomato DC3000 and P. syringae phaseolicola 1448A were searched using two programs: the PredictRegulon server 
[13] and the Virtual Footprint server [15]. Variations were made in the input sequence length and strand orientation. In Virtual Footprint predictions also different number of mismatches from the ColR binding consensus IUPAC code was allowed. Except for the parameters mentioned above, the programs were used with default settings. The new binding sites of $P$. putida ColR presented in this study were identified in a step-by-step process meaning that new predictions were made with every additional confirmed ColR binding site. Namely, first prediction with two input sequences (ColR sites in promoters of oprQ and PP0903) disclosed potential ColR binding sites in upstream regions of PP1636 and between divergently located PP0900 and PP0901 (colR). After experimental verification of ColR site between PP0900 and PP0901, the second round of prediction was performed with three input sequences resulting, for instance, in prediction of potential ColR sites upstream of PP0737 and PP0035. After verification of these sites, the third prediction was performed etc. Following such step-by-step process we were able to map seven ColR recognition sites, which were used as input in final prediction presented in Additional file 1.

\section{Authors' contributions}

PAK carried out in vitro binding experiments and most enzyme activity measurements, participated in the in silico analysis and drafted the first version of the manuscript. RK and $\mathrm{RH}$ constructed the promoter-probe plasmids and contributed to the enzyme measurements. MK participated in manuscript editing. RH conceived, designed and coordinated experimental work and manuscript editing. All authors read and approved the final manuscript.

\section{Additional material}

\section{Additional file 1}

Predicted regulon of ColR in P. putida. Output of PredictRegulon web server listing potential targets of response regulator ColR in $\mathrm{P}$. putida. Click here for file

[http://www.biomedcentral.com/content/supplementary/14712199-10-46-S1.doc]

\section{Additional file 2}

Potential ColR target genes in Pseudomonas fluorescens PfO-1. Virtual footprint predictions with IUPAC input sequences (YYVASDNYTTTTTSAC) and (GTSAAAAARNHSTBRR) in Pseudomonas fluorescens $P f O-1$ genome.

Click here for file

[http://www.biomedcentral.com/content/supplementary/14712199-10-46-S2.doc]

\section{Additional file 3}

Oligonucleotides. Sequences of oligonucleotides used in a current study. Click here for file

[http://www.biomedcentral.com/content/supplementary/14712199-10-46-S3.doc]

\section{Acknowledgements}

We are grateful to Tiina Alamäe, Marta Putrinš, Heili llves and Hanna Hõrak for critically reading the manuscript. This work was supported by grants 6025 and 7829 from the Estonian Science Foundation to R.H., by funding of Targeted Financing Project TLOMR003I from Estonian Ministry of Research and Education to M.K., and grant HHMI 550056I4 from the Howard Hughes Medical Institute International Research Scholars Program to M.K.

\section{References}

I. Stock AM, Robinson VL, Goudreau PN: Two-component signal transduction. Annu Rev Biochem 2000, 69:183-215.

2. Mascher T, Helmann JD, Unden G: Stimulus perception in bacterial signal-transducing histidine kinases. Microbiol Mol Biol Rev 2006, 70(4):910-938.

3. Robinson VL, Buckler DR, Stock AM: A tale of two components: a novel kinase and a regulatory switch. Nat Struct Biol 2000, 7(8):626-633.

4. Rodrigue A, Quentin Y, Lazdunski A, Mejean V, Foglino M: Twocomponent systems in Pseudomonas aeruginosa: why so many? Trends Microbiol 2000, 8(I I):498-504.

5. Pseudomonas Genome Database: A database for Pseudomonas aeruginosa and other Pseudomonas species genomes [http://www.pseudomonas.com]

6. Dekkers LC, Bloemendaal CJ, de Weger LA, Wijffelman CA, Spaink HP, Lugtenberg BJ: A two-component system plays an important role in the root-colonizing ability of Pseudomonas fluorescens strain WCS365. Mol Plant Microbe Interact 1998, II(I):45-56.

7. Hõrak R, Ilves H, Pruunsild P, Kuljus M, Kivisaar M: The ColR-ColS two-component signal transduction system is involved in regulation of Tn4652 transposition in Pseudomonas putida under starvation conditions. Mol Microbiol 2004, 54(3):795-807.

8. Kivistik PA, Putrinš M, Püvi K, llves H, Kivisaar M, Hõrak R: The CoIRS two-component system regulates membrane functions and protects Pseudomonas putida against phenol. J Bacteriol 2006, 188(23):8109-8II7.

9. Putrinš M, llves $H$, Kivisaar M, Hõrak R: ColRS two-component system prevents lysis of subpopulation of glucose-grown Pseudomonas putida. Environ Microbiol 2008, I O(I0):2886-2893.

10. Hu N, Zhao B: Key genes involved in heavy-metal resistance in Pseudomonas putida CD2. FEMS Microbiol Lett 2007, 267(I): 17-22.

II. de Weert S, Dekkers LC, Bitter W, Tuinman S, Wiifjes AH, van Boxtel R, Lugtenberg BJ: The two-component colR/S system of Pseudomonas fluorescens WCS365 plays a role in rhizosphere competence through maintaining the structure and function of the outer membrane. FEMS Microbiol Ecol 2006, 58(2):205-2।3

12. Predict Regulon Server [http://www.cdfd.org.in/predictregulon]

13. Yellaboina S, Seshadri J, Kumar MS, Ranjan A: PredictRegulon: a web server for the prediction of the regulatory protein binding sites and operons in prokaryote genomes. Nucleic Acids Res 2004:W3 18-320.

14. Virtual Footprint - bacterial regulon analyzer [http:// www.prodoric.de/vfp/]

15. Münch R, Hiller K, Grote A, Scheer M, Klein J, Schobert M, Jahn D: Virtual Footprint and PRODORIC: an integrative framework for regulon prediction in prokaryotes. Bioinformatics 2005, 2 I (22):4187-4189.

16. WebLogo - Create Sequence Logos [http://weblogo.berke ley.edu/logo.cgi]

17. de Been M, Bart MJ, Abee T, Siezen RJ, Francke C: The identification of response regulator-specific binding sites reveals new roles of two-component systems in Bacillus cereus and closely related low-GC Gram-positives. Environ Microbiol 2008, I0(10):2796-2809.

18. Trent MS, Pabich W, Raetz CR, Miller SI: A PhoP/PhoQ-induced Lipase (PagL) that catalyzes 3-O-deacylation of lipid A precursors in membranes of Salmonella typhimurium. J Biol Chem 200I, 276( I 2):9083-9092.

19. Rutten L, Geurtsen J, Lambert W, Smolenaers JJ, Bonvin AM, de Haan A, Ley P van der, Egmond MR, Gros P, Tommassen J: Crystal structure and catalytic mechanism of the LPS 3-O-deacylase PagL 
from Pseudomonas aeruginosa. Proc Natl Acad Sci USA 2006, I 03( I 8):707I-7076

20. Kawasaki K, China K, Nishijima M: Release of the lipopolysaccharide deacylase PagL from latency compensates for a lack of lipopolysaccharide aminoarabinose modification-dependent resistance to the antimicrobial peptide polymyxin B in Salmonella enterica. J Bacteriol 2007, I 89(I3):49| I-49|9.

21. Kawasaki K, Ernst RK, Miller SI: 3-O-deacylation of lipid A by PagL, a PhoP/PhoQ-regulated deacylase of Salmonella typhimurium, modulates signaling through Toll-like receptor 4 . J Biol Chem 2004, 279(1 9):20044-20048.

22. Zhang YM, Rock CO: Membrane lipid homeostasis in bacteria. Nat Rev 2008, 6(3):222-233.

23. Bayley SA, Duggleby CJ, Worsey MJ, Williams PA, Hardy KG, Broda $P$ : Two modes of loss of the Tol function from Pseudomonas putida mt-2. Mol Gen Genet 1977, 154(2):203-204.

24. J. Craig Venter Institute [http://www.tigr.org]

25. Miller $\mathrm{JH}$ : A short course in bacterial genetics: a laboratory manual and handbook for Echerichia coli and related bacteria. Cold Spring Harbour Laboratory Press, Cold Spring Harbour, NY; 1992.

26. Adams MH: Bacteriophages. Interscience Publishers Inc., New York; 1959.

27. Sharma RC, Schimke RT: Preparation of electrocompetent E. coli using salt-free growth medium. Biotechniques 1996, 20(I):42-44.

28. Tover A, Ojangu EL, Kivisaar M: Growth medium compositiondetermined regulatory mechanisms are superimposed on CatR-mediated transcription from the pheBA and catBCA promoters in Pseudomonas putida. Microbiology 200I, I47(Pt 8):2149-2156.

Publish with Biomed Central and every scientist can read your work free of charge

"BioMed Central will be the most significant development for disseminating the results of biomedical research in our lifetime. "

Sir Paul Nurse, Cancer Research UK

Your research papers will be:

- available free of charge to the entire biomedical community

- peer reviewed and published immediately upon acceptance

- cited in PubMed and archived on PubMed Central

- yours - you keep the copyright 